Research Paper

\title{
Characterization of Cancer Stem-Like Cells Derived from Mouse Induced Pluripotent Stem Cells Transformed by Tumor-Derived Extracellular Vesicles
}

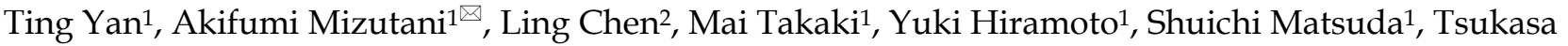 \\ Shigehiro ${ }^{1}$, Tomonari Kasai ${ }^{1}$, Takayuki Kudoh ${ }^{1}$, Hiroshi Murakami ${ }^{1}$, Junko Masuda ${ }^{1}$, Mary J. C. Hendrix ${ }^{3}$, \\ Luigi Strizzi³ ${ }^{3}$ David S. Salomon ${ }^{4}$, Li Fu' ${ }^{5}$, Masaharu Seno ${ }^{1 凶}$ \\ 1. Department of Biotechnology, Graduate School of Natural Science and Technology, Okayama University, 3.1.1 Tsushima-Naka, Kita-ku, Okayama \\ 700-8530, Japan. \\ 2. Department of Pathology, Tianjin Central Hospital of Gynecology Obstetrics, No. 156, Sanmalu, Nankai District, Tianjin, 300100, China. \\ 3. Lurie Children's Research Center, Feinberg School of Medicine, Northwestern University, 2300 Children's Plaza, Box 222, Chicago, IL 60614-3394, USA. \\ 4. Mouse Cancer Genetics Program, Center for Cancer Research, National Cancer Institute, Frederick, MD 2702, USA. \\ 5. State Key Laboratory of Breast Cancer Research, Department of Breast Cancer Pathology and Research Laboratory, Cancer Hospital of Tianjin Medical \\ University, Tianjin, China.
}

$\triangle$ Corresponding author: Akifumi Mizutani (e-mail: mizut-a@okayama-u.ac.jp, Phone/Fax +81-86-251-8209) and Masaharu Seno (e-mail: mseno@okayama-u.ac.jp, Phone/Fax +81-86-251-8216).

(c) Ivyspring International Publisher. This is an open-access article distributed under the terms of the Creative Commons License (http://creativecommons.org/ licenses/by-nc-nd/3.0/). Reproduction is permitted for personal, noncommercial use, provided that the article is in whole, unmodified, and properly cited.

Received: 2014.02.17; Accepted: 2014.05.21; Published: 2014.07.05

\begin{abstract}
Several studies have shown that cancer niche can perform an active role in the regulation of tumor cell maintenance and progression through extracellular vesicles-based intercellular communication. However, it has not been reported whether this vesicle-mediated communication affects the malignant transformation of normal stem cells/progenitors. We have previously reported that the conditioned medium derived from the mouse Lewis Lung Carcinoma (LLC) cell line can convert mouse induced pluripotent stem cells (miPSCs) into cancer stem cells (CSCs), indicating that normal stem cells when placed in an aberrant microenvironment can give rise to functionally active CSCs. Here, we focused on the contribution of tumor-derived extracellular vesicles (tEVs) that are secreted from LLC cells to induce the transformation of miPSCs into CSCs. We isolated tEVs from the conditioned medium of LLC cells, and then the differentiating miPSCs were exposed to tEVs for 4 weeks. The resultant tEV treated cells (miPS-LLCev) expressed Nanog and Oct3/4 proteins comparable to miPSCs. The frequency of sphere formation of the miPS-LLCev cells in suspension culture indicated that the self-renewal capacity of the miPS-LLCev cells was significant. When the miPS-LLCev cells were subcutaneously transplanted into Balb/c nude mice, malignant liposarcomas with extensive angiogenesis developed. miPS-LLCevPT and miPS-LLCevDT, the cells established from primary site and disseminated liposarcomas, respectively, showed their capacities to self-renew and differentiate into adipocytes and endothelial cells. Moreover, we confirmed the secondary liposarcoma development when these cells were transplanted. Taken together, these results indicate that miPS-LLCev cells possess CSC properties. Thus, our current study provides the first evidence that $\mathrm{tEV}$ s have the potential to induce CSC properties in normal tissue stem cells/progenitors.
\end{abstract}

Key words: cancer stem cells; mouse induced pluripotent stem cells; extracellular vesicles; cancerous niche; liposarcoma.

\section{Introduction}

The microenvironment/niche can exert profound genetic and/or epigenetic effects on stem cells

through interactions between stem cells and surrounding tissue resident cell populations, or through 
cell-derived factors originating from the surrounding cells within the niche. Current studies have demonstrated that an aberrant microenvironment with locally derived growth-promoting signals rather than growth-inhibiting signals may contribute to the genesis of cancer stem cells (CSCs) [1,2]. CSCs are defined as cells within a tumor that possess the capacity to self-renew and to give rise to the heterogeneous lineages of cancer cells that comprise the tumor [3]. Cells with CSC properties have been isolated from various types of tumors and cancer cell lines, including glioblastoma, lung carcinoma, Ewing's sarcoma, and breast cancer [4-7].

Despite many studies, the biological nature of CSCs remains largely unclear, especially the origin of CSCs. Findings pertinent to secreted exosome/microvesicle-based intercellular communication, has provided a potential mechanism involved in niche regulation of cell fate/behavior [8]. In this regard, tumor-derived extracellular vesicles (tEVs), which are mainly comprised of exosomes and microvesicles, are critical components in tumor niche that promote tumor progression [9]. Pivotal roles of microvesicles secreted by both human and murine lung cancer cell lines have been shown in tumor progression, metastasis and angiogenesis [10]. Exosomes that are derived from highly metastatic melanomas can also increase the metastatic behavior of primary melanomas through the education of bone marrow progenitor cells to create a pre-metastatic niche [11]. An oncogenic receptor, EGFRvIII, has been shown to be carried by microvesicles from aggressive glioma tumor cells to a non-aggressive tumor cell populations, and the recipient cells exhibited activation of MAPK and Akt signaling pathways, concomitant with a morphological transformation and an increase in anchorage-independent growth [12]. Microvesicles secreted by a human prostate cancer cells can activate normal stromal fibroblasts to cancer-associated fibroblasts, which is well known for contributing to tumorigenicity [13, 14]. In addition, adipose tissue-derived mesenchymal stem cells exhibit tumor-associated myofiblobrastic characteristics after treatment of exosomes that were derived from breast cancer cell lines [15]. These findings clearly indicate that tEVs act as messengers during cell-to-cell communication, which can significantly affect tumor progression and metastasis. To our knowledge, however, no direct report has demonstrated that tEVs can modulate the fate of normal stem cells or progenitor cells to facilitate their conversion or transformation into CSCs.

Since our previous study indicating that secreted factor(s) from various cancer cell lines could promote the formation of CSCs from miPSCs [16], it is highly possible that tEVs could be one mechanism underlying this conversion. In this study, we applied tEVs collected from LLC CM to differentiating miPSCs cultures, and characterized the resultant cells both in vitro and in vivo to assess the contribution of tEVs to induce CSCs from miPSCs. Our results suggested that normal stem cells or progenitor cells might give rise to CSCs when they are exposed to an abnormal cancerous niche. Understanding the mechanisms and details of this process will hopefully be useful in the development of new therapeutic approaches to target not only CSCs, but also the cancerous niche.

\section{Materials and Methods}

\section{Preparation and detergent treatment of tEVs from LLC cell line}

LLC cells at $80 \%$ confluence were cultured with serum-free Dulbecco's Modified Eagle Medium (DMEM). Culture supernatants were collected after 48 $\mathrm{h}$, then centrifuged at $300 \mathrm{~g}$ for $10 \mathrm{~min}$ and $2000 \mathrm{~g}$ for $10 \mathrm{~min}$ to remove cells and large debris, respectively. The cell-free supernatant was confirmed no cell contamination by incubation in cell culture incubator, then followed by centrifugation at $10,000 \mathrm{~g}$ for $30 \mathrm{~min}$ to remove small debris. tEVs were pelleted by ultracentrifugation (Himac CP70MXX, Hitachi, Japan) at 100,000 $\mathrm{g}$ for $2 \mathrm{~h}$, washed twice and suspended in PBS [17]. Particle diameter was measured by dynamic laser scattering (ELS-8000, Otsuka Electronics, Japan). Protein concentration was determined by MicroBCA Protein Assay kit (Pierce). tEVs were stored at $-80^{\circ} \mathrm{C}$ until use.

To disrupt the tEVs, $0.05 \mu \mathrm{g} / \mu \mathrm{L}$ tEVs were incubated with Triton X-100 at final concentration of $0.5 \%$ in $4^{\circ} \mathrm{C}$ on rotator.

\section{Cell culture}

Mouse iPSCs [18] that contained a puromycin (puro) resistant gene and green fluorescent protein (GFP) gene (iPS-MEF-Ng-20D-17, Lot No. 012, Riken Cell Bank, Japan) were maintained under the humidified $5 \% \mathrm{CO}_{2}$ atmosphere at $37^{\circ} \mathrm{C}$ on feeder layers of mitomycin-C-treated mouse embryonic fibroblasts (MEFs) (Reprocell, Japan) in miPS medium (DMEM containing $15 \%$ fetal bovine serum (FBS), $0.1 \mathrm{mM}$ Non-Essential Amino Acid (NEAA, Life Technologies), $2 \mathrm{mM}$ L-Glutamine, $0.1 \mathrm{mM}$ 2-mercaptoethanol, $1000 \mathrm{U} / \mathrm{mL}$ Leukemia inhibitory factor (LIF, Millipore), $50 \mathrm{U} / \mathrm{mL}$ penicillin and $50 \mathrm{U} / \mathrm{mL}$ streptomycin). Differentiated cells and MEFs were removed by culturing in the presence of $1 \mu \mathrm{g} / \mathrm{mL}$ puro. The Lewis Lung Carcinoma cell line (ATCC) was maintained in DMEM supplemented with $10 \%$ FBS.

For $\mathrm{tEV}$ treatment, miPSCs were first induced to 
differentiate for 3 days by culturing without LIF. Then, $4 \times 10^{5}$ cells / 60 -mm dish differentiating miPSCs were maintained in miPS medium (without LIF) containing various concentrations of LLC $\mathrm{tEVs}$, and medium was changed daily with fresh $\mathrm{tEVs}$ or detergent pre-treated tEVs. When cells reached $80 \%$ confluence, they were harvested and seeded in the corresponding medium as the number of $4 \times 10^{5}$ cells/ $60-\mathrm{mm}$ dish. The resultant cells (miPS-LLCev) were maintained with miPS medium without LIF (Fig. 1A).

For suspension culture, $4 \times 10^{4}$ single cells were plated in $60-\mathrm{mm}$ Lipidure ${ }^{\circledR}$-coated low adhesion dishes (NOF Corporation, Japan) in serum-free miPS medium without LIF, but supplemented with Insulin-Transferrin-Selenium-X (ITS-X, Life Technologies). Spheroids with diameters above $100 \mu \mathrm{m}$ were counted under a fluorescence microscope (Olympus IX81, Japan) on day 4. After image capturing on day 7, spheres were collected for further analyses.

For adipocyte differentiation, spheroids of tumor cells were directly seeded under adherent conditions in miPS medium (without LIF). After growing to $90 \%$ confluence, the medium was changed to adipose differentiation medium (miPS medium (without LIF), supplemented with $10 \mu \mathrm{g} / \mathrm{mL}$ insulin and $2.5 \mu \mathrm{M}$ dexamethasone (AdipoInducer, ReagentTakara, Japan)) for 2 days. Subsequently, the cells were maintained in miPS medium (without LIF) supplemented with $10 \mu \mathrm{g} / \mathrm{mL}$ insulin for another 6 days, followed by Oil Red $\mathrm{O}$ staining to confirm the adipogenesis.

\section{Tumorigenicity analysis in immunodeficient mice}

4 week-old Balb/c-nu/nu female immunodeficient mice were purchased from Charles River (Japan). $1 \times 10^{2}-1 \times 10^{6}$ Cells (shown in Table 1) were suspended in sterile PBS and subcutaneously injected into one flank of immunodeficient mice. After 4 weeks, all tumors were resected at autopsy and sectioned for histologic analysis. All animal experiments were reviewed and approved by the ethics committee for animal experiments of Okayama University under the ID OKU-2013252.

\section{Immunohistochemistry (IHC) and Oil Red O staining}

IHC staining was performed as previously described [16] by using rat monoclonal anti-CD31 antibody (1:200, Santa Cruz), rabbit polyclonal anti-Ki67 antibody (1:200, Abcam), rabbit monoclonal anti-GFP antibody (1:300, Cell Signaling), mouse monoclonal anti-vimentin antibody (1:100, Santa Cruz), or mouse monoclonal anti-PPAR 2 antibody (1:200, Santa Cruz).
For Oil Red O staining, cells or $10 \mu \mathrm{m}$ cryosections were fixed by neutral buffered $10 \%$ formalin, 30 $\mathrm{min}$ at room temperature. After rinsing in $60 \%$ isopropanol, cells were stained with Oil Red O (Sigma) at $60^{\circ} \mathrm{C}$ for $5 \mathrm{~min}$.

\section{Immunoblotting and immunofluorescence}

Thirty-five micrograms of total cellular protein of each sample was loaded for electrophoresis. Separated proteins were then blotted onto polyvinylidene difluoride (PVDF) membrane (Millipore). After blocking for $1 \mathrm{~h}$ with $5 \%$ skim milk, the membranes were incubated for $12 \mathrm{~h}$ at $4^{\circ} \mathrm{C}$ with primary antibodies: rabbit polyclonal anti-CD63 antibody (1:1000, Santa Cruz), rabbit polyclonal anti-Nanog antibody (1:3000, Abcam) or mouse monoclonal anti-Oct3/4 antibody (1:3000, Santa Cruz). The blots were then incubated with secondary antibody, either horseradish peroxidase (HRP)-conjugated anti-rabbit or anti-mouse IgG (Cell Signaling). The bound antibodies were detected using Western Lighting plus-ECL reagent (PerkinElmer), and recorded by Light-Capture II cooled CCD camera system (ATTO, Japan). Coomassie blue staining of SDS-PAGE gels or anti- $\beta$-actin (Cell Signaling) immunoblotting were used as controls for equal loading. Semi-quantitation of the bands was performed using ImageJ software (NIH, MD) according to the instruction.

Table I. The tumorigenic potential of miPS-LLCev cells within one month.

\begin{tabular}{llll}
\hline Samples & No. of cells & $\begin{array}{l}\text { Tumor } \\
\text { formation }\end{array}$ & Histologic examination \\
\hline miPS-LLCev & $1 \times 10^{2}$ & $0 / 4$ & NA \\
& $1 \times 10^{3}$ & $0 / 4$ & NA \\
& $1 \times 10^{4}$ & $0 / 4$ & NA \\
& $1 \times 10^{5}$ & $3 / 4$ & malignant, angiogenesis \\
& $1 \times 10^{6}$ & $10 / 10^{*}$ & malignant, angiogenesis, \\
miPS-LLCevPT & $1 \times 10^{2}$ & $0 / 4$ & NA \\
& $1 \times 10^{3}$ & $0 / 4$ & NA \\
& $1 \times 10^{4}$ & $0 / 4$ & NA \\
& $1 \times 10^{5}$ & $5 / 5$ & malignant, angiogenesis \\
& $1 \times 10^{6}$ & $5 / 5$ & malignant, angiogenesis \\
miPS-LLCevDT & $1 \times 10^{2}$ & $0 / 4$ & NA \\
& $1 \times 10^{3}$ & $0 / 4$ & NA \\
& $1 \times 10^{4}$ & $0 / 4$ & NA \\
& $1 \times 10^{5}$ & $5 / 5$ & malignant, angiogenesis \\
& $1 \times 10^{6}$ & $5 / 5$ & malignant, angiogenesis \\
\hline
\end{tabular}

PT: primary tumor cells; DT: disseminated tumor cells.

NA: not applicable.

*There are 2 in 10 mice were found to be bleeding with disseminated tumors in the mesentery. 
A



\begin{tabular}{|l|l|}
$\begin{array}{l}1 \text { week } \\
\text { self-renewal }\end{array}$ & $\begin{array}{l}3 \text { days } \\
\text { differentiation }\end{array}$ \\
\hline
\end{tabular}

B

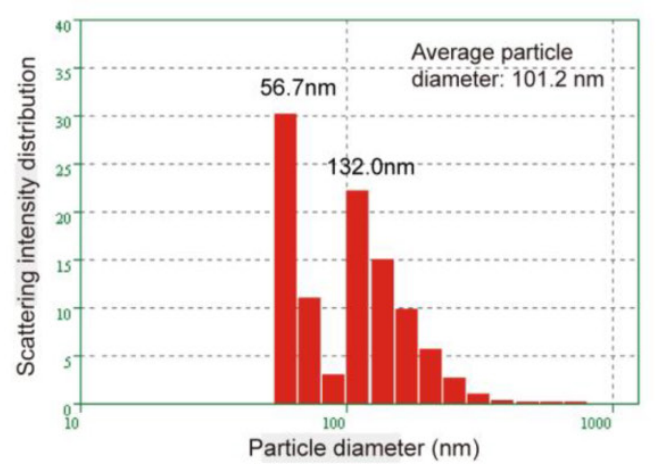

D
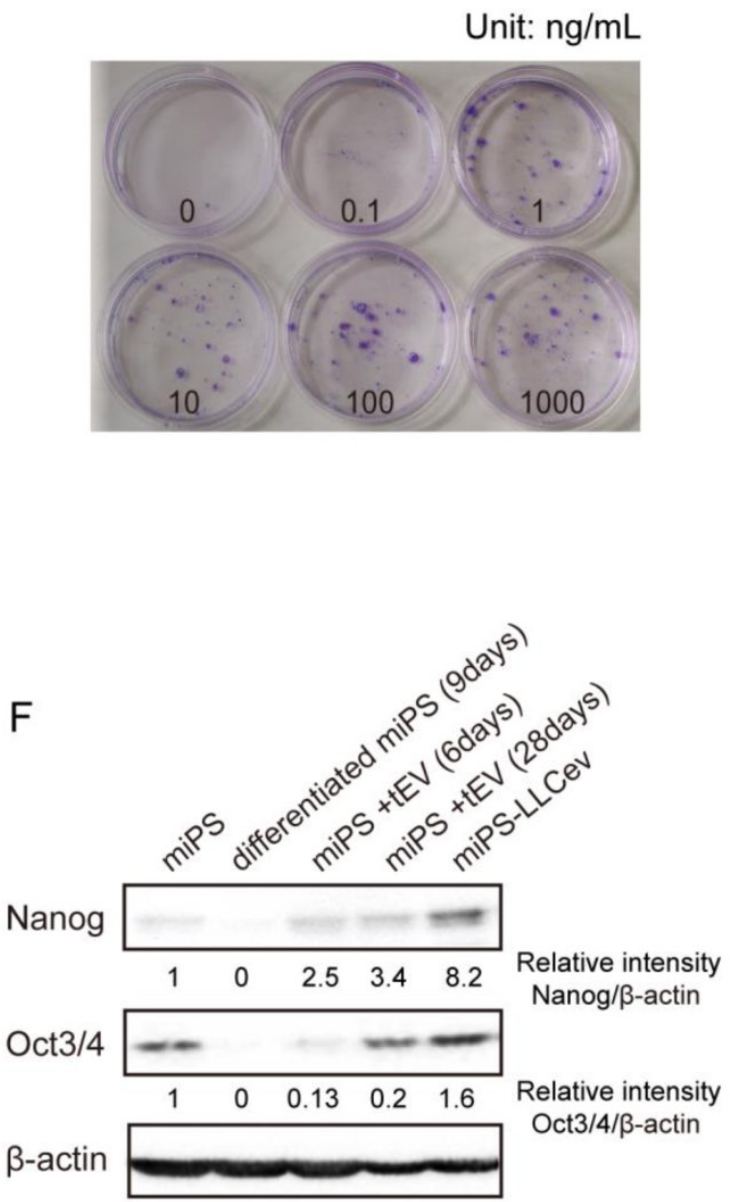

C

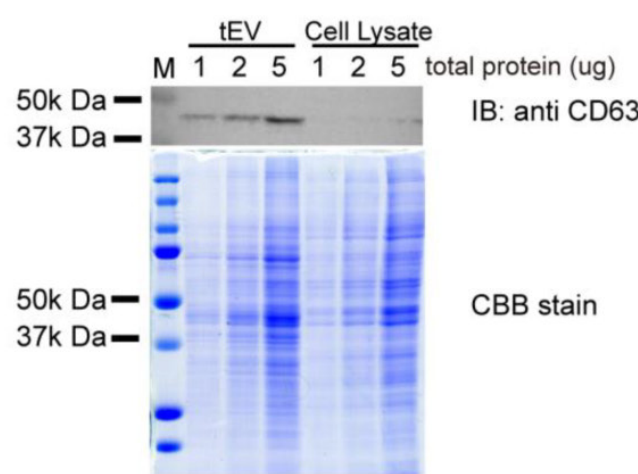

E

+tEV (100ng/mL)

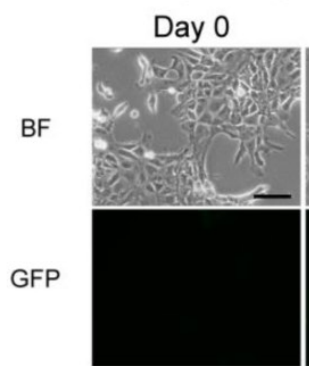

3 weeks
1 week

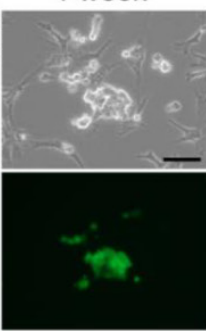

4 weeks

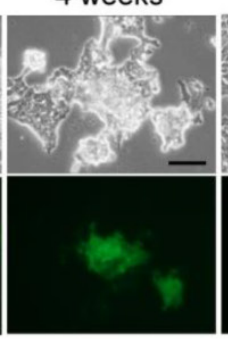

-tEV

Day 0

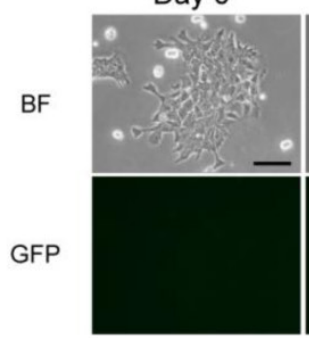

GFP

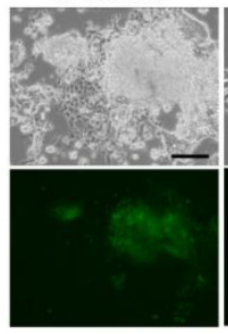

1 week

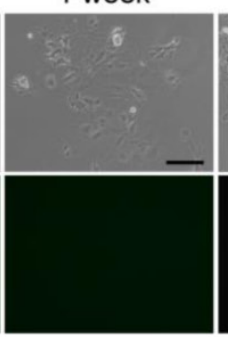

2 weeks

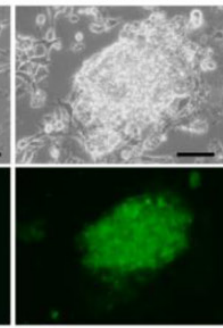

miPS-LLCev

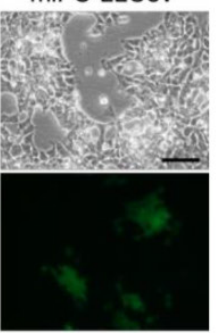

2 weeks

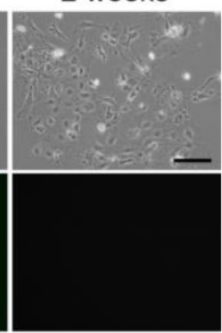

Figure I. tEV treatment of differentiating miPSCs gives rise to stem-like cells. (A) Cells are passaged following the conversion schedule. Each color indicates different culture media. (B) Size distribution of tEVs collected from LLC CM. (C) Immunoblotting analysis of CD63 in tEVs and LLC cell lysates shows the enrichment of exosomes. Coomassie stain of SDS-PAGE gel shows equal loading of total protein. (D) Colony formation in indicated concentrations of tEVs cultured for 2 weeks. (E) Cell images during conversion by $100 \mathrm{ng} / \mathrm{mL}$ tEV are shown. Cells passaged in plain medium (-tEV) are used as control. Scale bar: I00 $\mu \mathrm{m}$. (F) Immunoblotting analysis of Nanog and Oct3/4 in the total protein from miPSCs (miPS), differentiated miPSCs (differentiated miPS (9days)), differentiated cells by tEVs cultured for 6 days (miPS + tEV (6days)), differentiated cells by tEVs cultured for 28 days (miPS + tEV (28days)) and resultant cells (miPS-LLCev). Relative intensities are normalized to that of $\beta$-actin. 
For immunofluorescence analysis, cells were deposited on coverslips, fixed with $4 \%$ paraformaldehyde, permeabilized with $0.1 \%$ Triton- $X$, blocked with 5\% BSA, incubated with rabbit polyclonal anti-CD34 antibody (1:200, Santa Cruz), rabbit polyclonal anti-CD36 antibody (1:200, Santa Cruz), mouse monoclonal anti-PPARY2 antibody and TexasRed-conjugated secondary antibodies and then counterstained with DAPI (Vector Laboratories). Images were captured using a confocal microscope equipped with a light fluorescence device (LSM510META, Carl Zeiss, Germany).

\section{Quantification of gene expression by quantitative reverse-transcription $\mathbf{P C R}$ (qRT-PCR)}

Total RNA was prepared from cells with RNeasy kit (Qiagen) and treated with DNase I (Takara) to remove any residual genomic DNA, then reverse transcribed by SuperScript III Reverse Transcriptase (Invitrogen). qRT-PCR was performed with LightCycler 480 SYBR Green I Master mix (Roche). Primer sequences are described in Additional File 1: Supplementary Table S1. Relative gene expression was normalized to that of Gapdh. All the experiments were repeated at least three times. Results are presented as means \pm SD. Student $t$-test was used to compare the expression of genes between unique sample and parental miPSCs. One-way ANOVA followed by post hoc Tukey analysis was used to assess the significance of the genes expression between miPS-LLCev, 'spheroids of bulk cell' and 'spheroids of puro resistant cell'. $P<0.05$ was considered significantly different.

\section{In vitro tube formation assay}

Individual cells were suspended in endothelial basal medium supplemented with FBS, hydrocortisone, hFGF-B, VEGF, R33-IGF-1, ascorbic acid, hEGF, GA-1000 and heparin (EGM-2 Single Quots Kit, Takara) following manufacture's instruction and seeded in triplicate on Matrigel (Becton Dickinson) coated chamber slides (Nunc) [19]. After 24 hours, cells were stained with fluorescence labeled rat anti-CD31 antibody.

\section{Invasion assay}

The Matrigel invasion assay was performed according to the manufacturer's protocol (Becton Dickinson). The growth factor-reduced Bio-coat Matrigel membrane inserts were rehydrated with serum-free medium and incubated at $37^{\circ} \mathrm{C}$ for $2 \mathrm{~h}$. Following aspiration of the medium from the inserts, $2.5 \times 10^{4}$ cells in $500 \mu \mathrm{L}$ serum-free medium were seeded onto the insert, and $750 \mu \mathrm{L}$ serum containing medium was added to the bottom well of the insert. After incuba- tion for $30 \mathrm{~h}$ in $5 \% \mathrm{CO}_{2}$ atmosphere at $37^{\circ} \mathrm{C}$, cells on the bottom of the membranes were fixed and counted after Gimsa staining.

\section{Results}

\section{tEV treatment of differentiating miPSCs gives rise to stem-like population of cells}

To more fully define the particular fraction in $\mathrm{CM}$ that can enhance CSC generation from miPSCs of previous study [16], we assessed the contribution of tEVs to this process in the current study. We isolated tEVs from LLC CM using standard method. The size distribution was quantified by dynamic laser scattering with an average size of $101.2 \mathrm{~nm}$ (Fig.1B). In addition, the presence of an exosomal marker, CD63 was identified [20] (Fig.1C). Intriguingly, the particle size distribution of the collected fractions indicated two peaks (56.7 $\mathrm{nm}$ and $132.0 \mathrm{~nm}$ ), suggesting that LLC cells secrete two different size populations of particles [17]. We conclude that the precipitated fraction contains exosomes and microvesicles, as judged from their size distribution and detection of CD63 [17, 20].

We then investigated whether $\mathrm{tEVs}$ were effective on promoting the conversion of differentiating miPSCs into CSCs. A schematic overview of the conversion procedure is illustrated in Figure 1A. The differentiating miPSCs were cultured in the medium containing purified tEVs for 4 weeks. The resultant cells, named miPS-LLCev, were maintained in miPS medium without LIF. The selection of undifferentiated miPSCs and monitoring of differentiation could be carried out by addition of puro and assessment of GFP fluorescence, respectively, since the expression of the puro resistant gene and GFP gene were under the control of the mouse Nanog promoter in the miPSCs used here [18].

To evaluate the optimal concentration of tEVs that can facilitate GFP positive colony formation, we tested different concentrations of tEVs $(0-1000 \mathrm{ng}$ protein of $\mathrm{tEVs} / \mathrm{mL}$ ) in culture medium. After 2 weeks, the number of colonies increased in a dose dependent fashion, and the most efficient cell growth was observed at $100 \mathrm{ng}$ of $\mathrm{tEVs} / \mathrm{mL}$. Under this concentration, cells grew actively and lead to a rapid colony expansion (Fig. 1D). Considering the recovery of tEVs from CM, this concentration is similar to that in the CM used for CSC-conversion (150 250 ng/mL) [16]. Therefore, we used $100 \mathrm{ng} / \mathrm{mL}$ of $\mathrm{tEVs}$ as the optimal condition for conversion of CSCs. To define more clearly the relevance of $\mathrm{tEVs}$ to the colony formation, we tested the detergent pre-treated $\mathrm{tEVs}$, whose membrane particle structure were disrupted. We observed that the number and size of colonies were significantly reduced (Additional File 1: Sup- 
plementary Fig. S1), which suggesting that the factor(s) responsible to the CSC-conversion were delivered from cancer cells as a form of membrane particles, tEVs.

During the 3 days culturing of miPSCs in the absence of LIF (Day -3 to Day 0), GFP expression, which is an index of Nanog expression, gradually decreased (Fig 1E, Day 0). In the following two weeks without tEV treatment, there were no GFP positive cells left and the number of surviving cells decreased in sequential passages. The cells acquired an enlarged and flattened morphology, indicating normal differentiation of miPSCs (Fig. 1E, -tEV). In contrast, with treatment of $100 \mathrm{ng} / \mathrm{mL}$ of tEVs, GFP positive colonies re-appeared within a week and grew during the conversion period (Fig. 1E, +tEV). These colonies were intermixed with GFP negative cells, which were morphologically distinct from the normal differentiated cells. No GFP positive cells were observed in the absence of tEVs after 2 weeks.

The re-emergence of GFP positive cells in the conversion medium suggested the acquisition of an embryonic stem (ES)-like phenotype in these cells, since the GFP gene is located downstream of the Nanog promoter, which is activated in ES cells and aggressive tumors $[18,21]$. We confirmed the increase in expression of Nanog and Oct3/4 by immunoblotting analysis during this transition phase (Fig. 1F). Compare to 9 days differentiated cell, the amounts of Nanog and Oct3/4 in the counterpart that treated by tEVs were increased. The amout of Nanog protein in miPS-LLCev were significantly higher than those in parental miPSCs. These results suggested that treatment with $\mathrm{tEV}$ s derived from LLC cells could give rise to a stem-like population from differentiating miPSCs by interfering with a normal differentiation program in these cells. This reprograming process might be important in the re-acquisition of a more embryonic stem-like phenotype. However, we cannot exclude the possibility that tEVs may directly maintain the undifferentiated state of miPSCs even though these cells are exposed to a differentiation competent environment [22].

\section{ES cell marker expression and self-renewal capacity of miPS-LLCev cells}

Considering the arise of stem-like cells and the results of our previous study [16], we expected that secreted $\mathrm{tEV}$ was one of the responsible factors that might contribute to the conversion of miPSCs into CSCs. Thus, we investigated the CSC properties of the miPS-LLCev cells. The self-renewal capacity of miPS-LLCev cells was assessed in the non-adherent condition, since malignant stem-like cells have been shown to form spheroids indicating self-renewal ca- pacity in suspension culture [23]. Approximately $12 \%$ of the bulk-unselected populations in miPS-LLCev cells expressed GFP. The large population of low GFP expressing miPS-LLCev cells prompted us to concentrate on GFP positive cells as puro resistant cells (Fig. 2A). We assessed the puro resistant GFP positive cells for growth in suspension culture, and found that the GFP positive population could form spheroids (Fig. 2B). All of the resultant spheroids were GFP positive, demonstrating self-renewal capacity of the GFP positive cells. To our surprise, the bulk population cells of miPS-LLCev that were not selected for puro resistance also formed spheroids, and only $17.8 \%$ of spheroids were expressing GFP (Fig. 2B and C).

By qRT-PCR analysis, we assessed the expression of genes, such as Oct3/4, Sox2, Klf4 and c-Myc, which perform a dominant role in ES and iPSC maintenance, self-renewal and reprograming (Fig. 2D). The level of each endogenous gene and total mRNAs (endogenous + transgene) expression were confirmed by using specific primers [24]. We did not detect any aberrant activation of trensgenes, pertinent to viral-transduction for the establishment of miPSCs (Additional File 1: Supplementary Fig. S2). In spheroids derived from the bulk of miPS-LLCev cells, Sox2 (6-fold in total and 2.5-fold in endogenous) and Klf4 (14-fold in total and 5-fold in endogenous) expression were significantly elevated compare to miPSCs, spheroids derived from puro resistant cells expressed higher levels of Sox2 (3.5-fold in total) and Klf4 (5.4-fold in total and 2-fold in endogenous) compared with miPSCs, but less than those of bulk spheroids. It has been reported that a narrow range of the amount of Oct $3 / 4$ is critical for maintaining pluripotency [25, 26]. In the spheroids derived from either puro resistant cells or bulk cells, Oct 3/4 was found to be up-regulated when compared with the miPSCs. The expression of this gene in the puro resistant GFP positive spheroids was significantly higher (1.7-fold in total and 1.9-fold in endogenous) than that in bulk spheroids $(P<0.05)$, implicating a potentially more stem-like state compared with GFP negative spheroids. These results indicate the potential presence of a subpopulation that possesses self-renewal capacity in the total miPS-LLCev cell population. Additionally, the bulk miPS-LLCev cell population contains a hierarchy in vitro ranging from $\operatorname{Nanog}^{+}\left(\mathrm{GFP}^{+}\right) / O c t 3 / 4^{+}$ stem-like cells to more differentiated cells. The elevation of Sox 2 and Klf4 might be related not only to the maintenance of the stem cell-like state, but also to unipotent differentiation potential.

\section{Generation of liposarcoma-like tumors in immunodeficient mice by miPS-LLCev cells}

It is known that, miPSCs generated teratomas 
after transplanted into nude mice [16, 18]. Unlike miPSCs, malignant-tumorigenic potential is an established property for the assessment of CSC activity [3]. To test this, $1 \times 10^{2}-1 \times 10^{6}$ miPS-LLCev bulk cells were subcutaneously injected into immunodeficient mice (Table 1). Tumors that developed in immunodeficient mice after 4 weeks were histologically and immunohistochemically analyzed (Fig. 3). A substantial portion of the tumor expressed a malignant phenotype, such as high nuclear to cytoplasmic ratio, a high mitotic rate and nuclear pleomorphism, and elevated expression of Ki-67, which are all properties indicative of a neoplastic state. Also we found anti-CD31 staining in the tumor, indicating tumor angiogenesis. We then assessed expression of vimentin and Pan-Cytokeratin for classifying the type of tumor. In the region of tumor where GFP expression was negative, vimentin staining was observed to be expressed in a diffuse pattern. Although we observed a CK-positive area in the tumor (data not shown), we concluded that the tumors were sarcomas. The GFP and vimentin staining patterns indicated the heterogeneity of cells in these sarcomas in the context of mesenchymal differentiation.
A

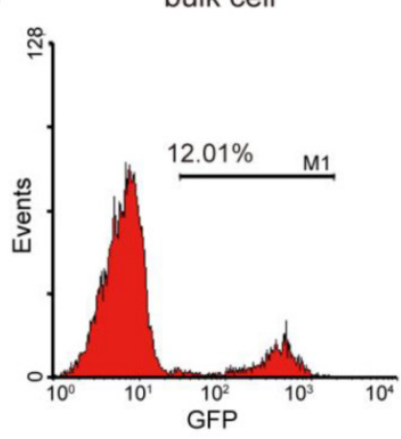

puro selected

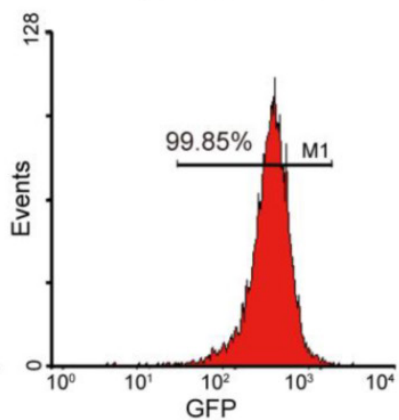

B

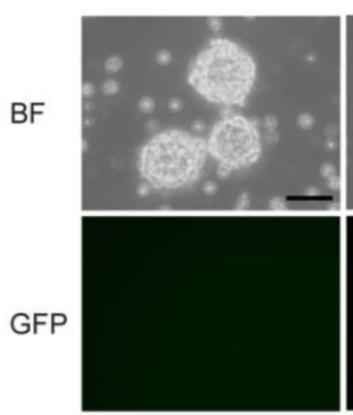

puro selected

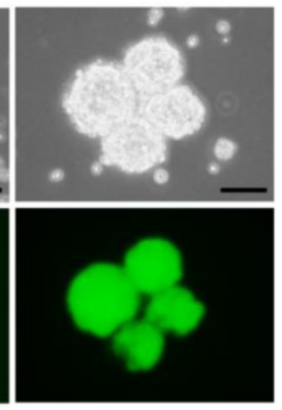

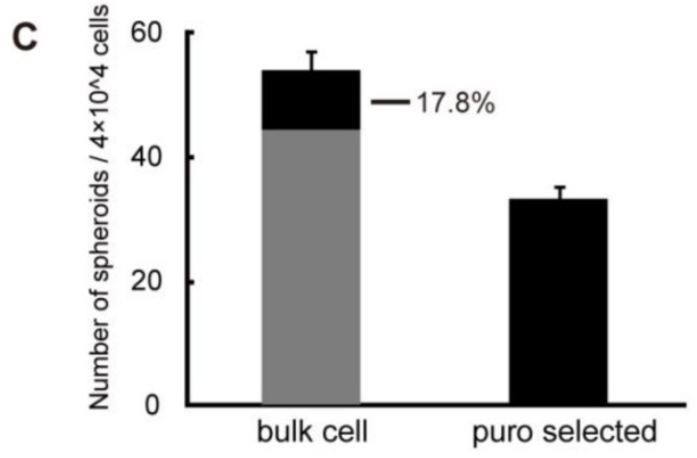

D

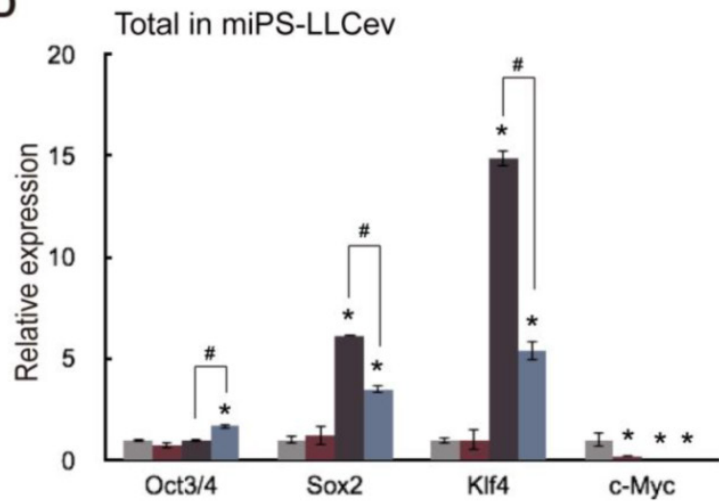

GFP- spheroids

GFP+ spheroids
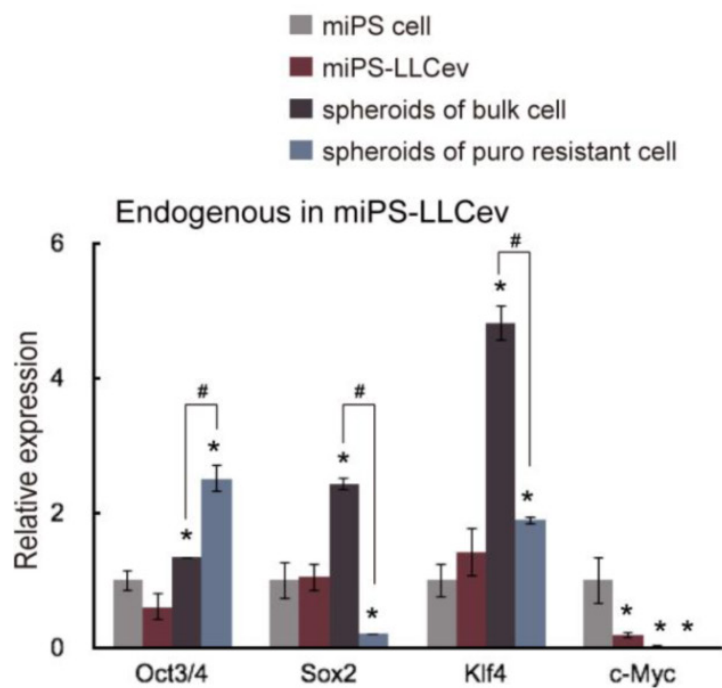

Figure 2. miPS-LLCev cells possess self-renewal capacity. (A) FACS analysis shows GFP population in bulk and puro selected miPS-LLCev cells. (B) Sphere formation assay in serum-free medium shows spherogenic potential of both GFP negative (bulk cell) and GFP positive (puro selected) miPS-LLCev cells. Scale bars: I00 $\mu$ m. (C) $4 \times 10^{4}$ bulk or puro selected single cell suspension were applied to serum-free condition, the number of GFP+ and GFP- spheroids above $100 \mu \mathrm{m}$ were scored after 4 days. The assay was performed in triplicate and the error bars indicate the difference in the total number. (D) qRT-PCR analysis of four transcription factors Oct3/4, Sox2, KIf4 and c-Myc expression levels in the indicated samples. The products of coding regions and endogenous transcripts are regarded as total and endogenous. Relative expression values are normalized to Gapdh and conducted in triplicate. Student t-test was used to compare the expression level between unique sample and miPSCs. Each asterisk shows the significance of $P<0.05$. One-way ANOVA followed by post hoc Tukey analysis was used to assess the significance of the genes expression between miPS-LLCev, 'spheroids of bulk cell' and 'spheroids of puro resistant cell'. Each '\#' shows the significance of $P<0.05$. 

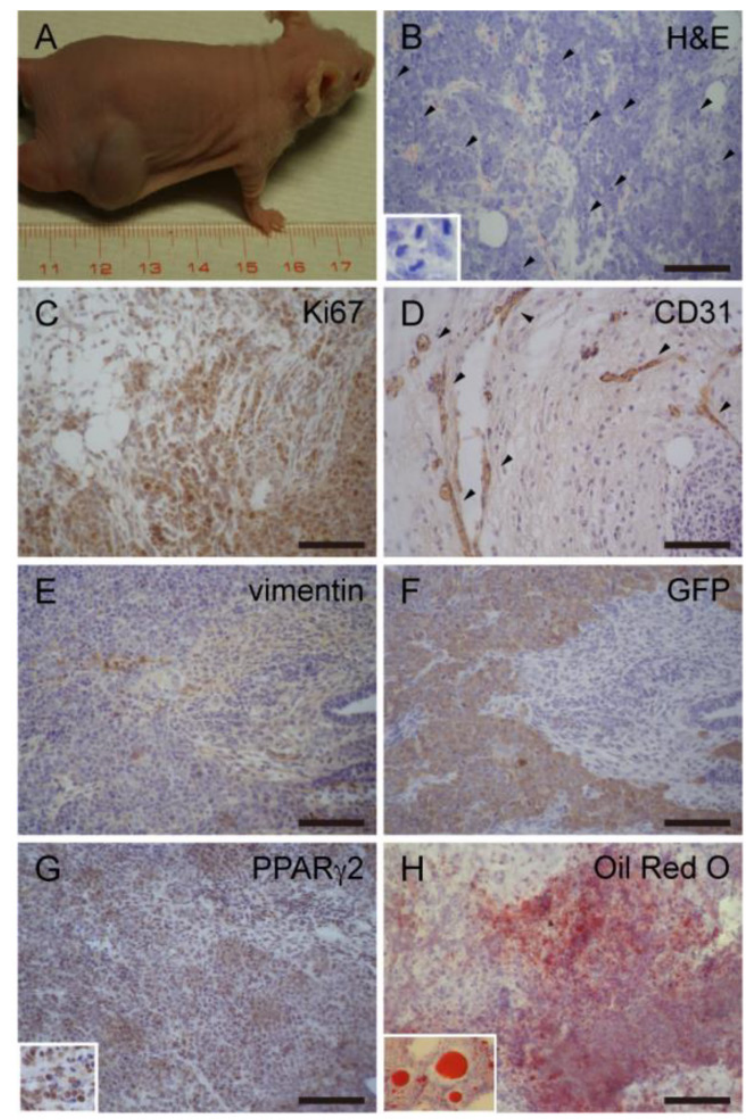

Figure 3. miPS-LLCev generate liposarcoma-like tumors in immunodeficient mice. (A) Generation of tumors after subcutaneous injection shows rapid growth of tumors. (B) H\&E staining of primary tumors dissected from injection site, showing multiple pathologic mitotic figures (arrowheads, inset) and hyper-vascularization. (C) IHC staining of Ki67 shows cellular proliferation. (D) IHC staining of $C D 31$ shows angiogenesis in tumors (arrowheads). Serial sections were stained with vimentin (E) and GFP (F), diffuse staining of vimentin is detected with opposite pattern to GFP in tumor. (G) IHC staining of PPAR $\gamma 2$ shows a large population of PPAR $\gamma 2$ expressing cells. $(\mathrm{H})$ Oil Red $\mathrm{O}$ staining shows fat droplets in tumors. Scale bars: $100 \mu \mathrm{m}$

It is worthwhile to note that there was a substantial proportion of adipose tissue in the tumors. We then investigated the expression of PPAR 2 , which is a nuclear hormone receptor that performs a critical role in regulating adipocyte differentiation [27]. Numerous cells were positive for PPARy2, suggesting differentiation into an adipocyte lineage. Furthermore, Oil Red O staining of cryosections revealed the presence of lipid droplets in the tumors. Therefore, tumors derived from miPS-LLCev cells are probably liposarcoma [28, 29]. Additionally, during tumor development, we observed some animals with abdominal bleeding, were bearing disseminated tumor nodules in the mesentery (Additional File 1: Supplementary Fig. S3 A and B).

\section{Self-renewal potential of cells derived from miPS-LLCev tumors}

To further evaluate the properties of miPS-LLCev cells as potential CSCs, we established cell lines from both the primary tumors (miPS-LLCevPT) and disseminated tumor nodules (miPS-LLCevDT), respectively (Additional File 1: Supplementary Fig. S3). In contrast to the original miPS-LLCev cells in vitro, the populations of GFP positive cells in both the miPS-LLCevPT and miPS-LLCevDT cell lines was significantly higher (73.39\% and $72.93 \%$, respectively) (Fig. $4 \mathrm{~A}$ ). In suspension culture, both cell lines were able to form spheroids indicating self-renewal capacity, and all spheroids were GFP positive (Fig. 4B and C). The expression levels of Oct3/4 in miPS-LLCevPT and miPS-LLCevDT, both in adherent and suspension cultures, were comparable to those in miPSCs (Fig. 4D). In contrast, significant higher levels of Sox2 and Klf4 expression were observed in the spheroids from both cell lines. We confirmed that there was no aberrant expression of the Yamanaka transgenic factors in either cell line (Additional File 1: Supplementary Fig. S2). $c-M y c$ was not expressed aberrantly in any of the cells suggesting a negligible contribution of this gene to the transformation and/or tumorigenicity in these cells. However, endogenous Nanog, Sox 2 and Klf4 might contribute to these properties [21, 30-32].

When the miPS-LLCevPT and miPS-LLCevDT cells were injected into immunodeficient mice, malignant liposarcomas again developed (Table 1). The pathological and immunohistochemical features of these secondary tumors were similar to the primary tumors indicating the repeatable nature of tumorigenesis in this model (Fig. 5). Collectively, we conclude that the miPS-LLCevPT and miPS-LLCevDT cells were able to maintain a self-renewal capacity and the original histotype of the primary tumor.

\section{The heterogeneity of tumor derived cells}

Distinct stages of adipogenesis and immunophenotypic plasticity have demonstrated a cellular hierarchy in human liposarcomas, the expression of both CD34 and CD36 could serve as specific markers indicative of adipose differentiation [33]. We then assessed the cellular lineage of adipocytes in the miPS-LLCevPT and miPS-LLCevDT cells in vitro. Immunostaining against CD34, which is a multipotent progenitor marker, expressed by adipose progenitors and other progenitor cell types [33-35], showed that both the miPS-LLCevPT and miPS-LLCevDT cells contained a population of CD34 expressing cells (Fig. $6 \mathrm{~A})$. There were heterogeneous populations within both cell lines exhibiting $\mathrm{GFP}^{+} / \mathrm{CD} 34^{+}, \mathrm{GFP}^{-} / \mathrm{CD} 34^{+}$ and $\mathrm{GFP}^{+} / \mathrm{CD} 34^{-}$. In the case of CD36 and PPARy2 expression, similar to $\mathrm{CD} 34$, there were positive and negative cells in both the GFP ${ }^{+}$and GFP- cells for these additional adipocyte markers (Fig. 6B and C). 
A
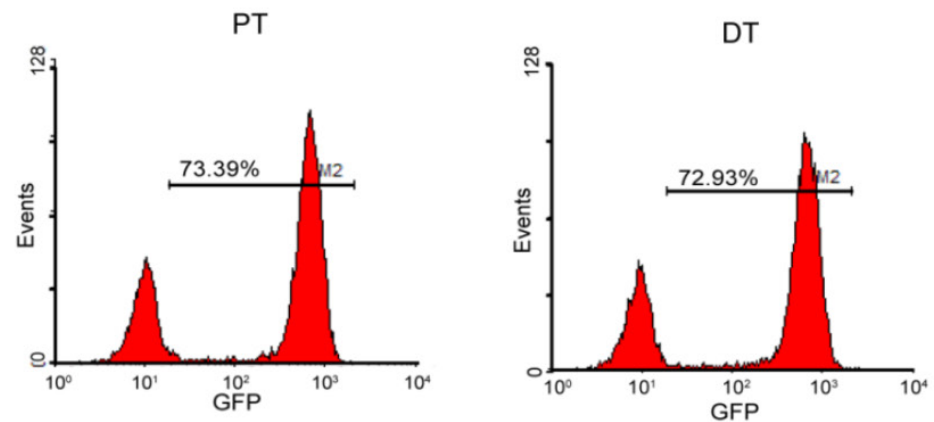

B

PT

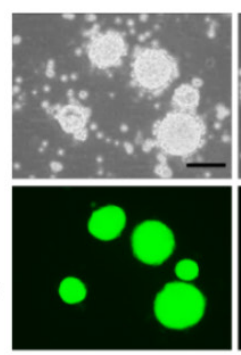

D
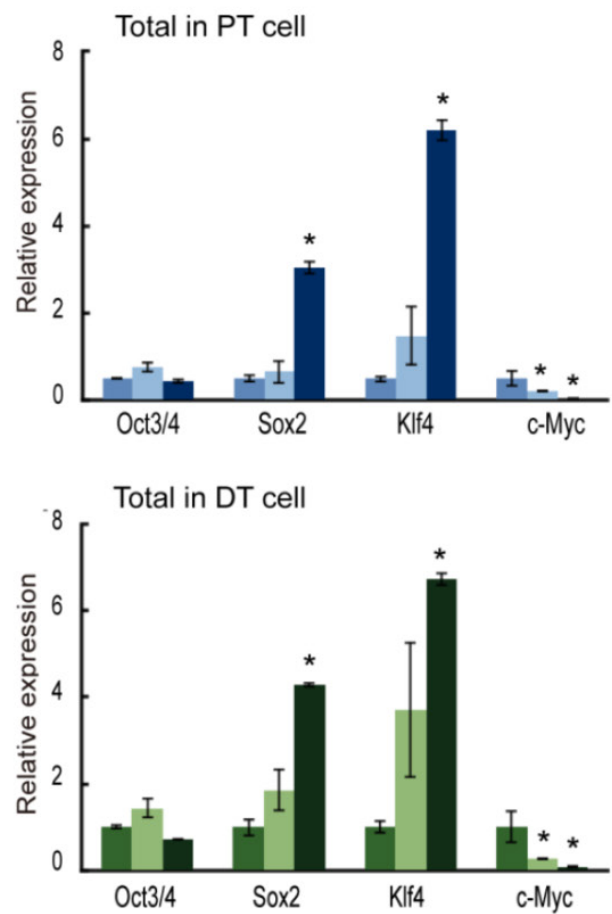

DT
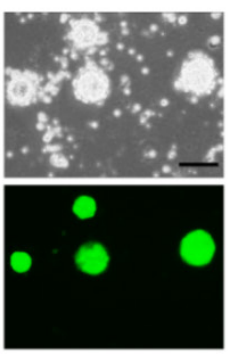

C

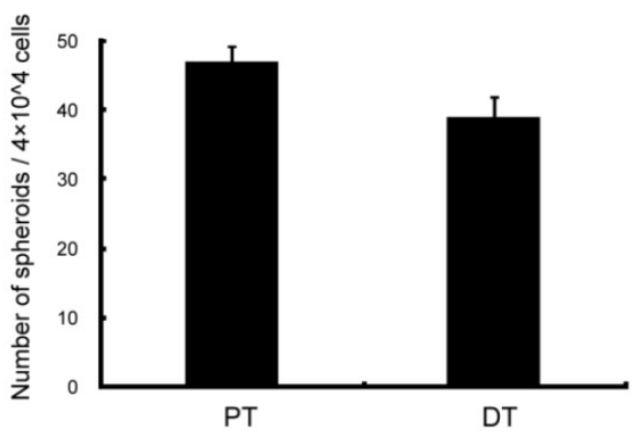

Endogenous in PT cell

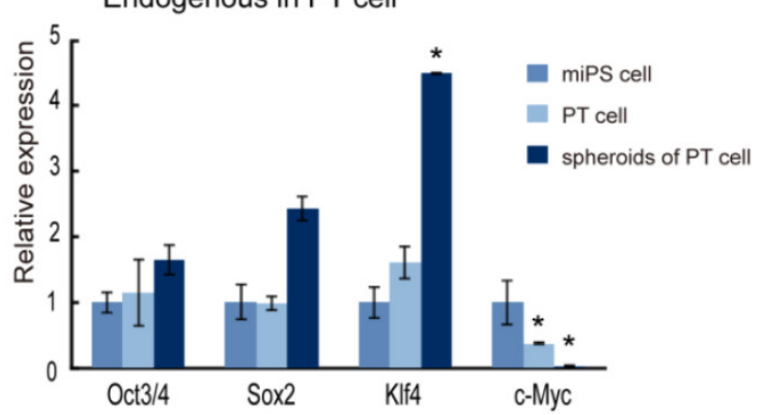

Endogenous in DT cell



Figure 4. The primary cells show self-renewal capacity. (A) FACS analysis of GFP in both primary cell lines shows GFP population. (B) Both primary bulk cells are able to form spheroids in suspension culture, and all the spheroids are GFP positive. (C) The number of spheroids (above $100 \mu \mathrm{m}$ ) from $4 \times 10^{4}$ bulk primary cells. Assays were performed in triplicate and the error bars indicate the difference in the total number. (D) qRT-PCR analysis of four transcription factors Oct3/4, Sox2, KIf4 and c-Myc expression levels in the indicated samples, which according to miPS-LLCev cell, express high level of Sox 2 and KIf4 in spheroid growing cells. Each asterisk shows the significance of $P<0.05$. Scale bars: $100 \mu \mathrm{m}$. 
Tumor from PT cell
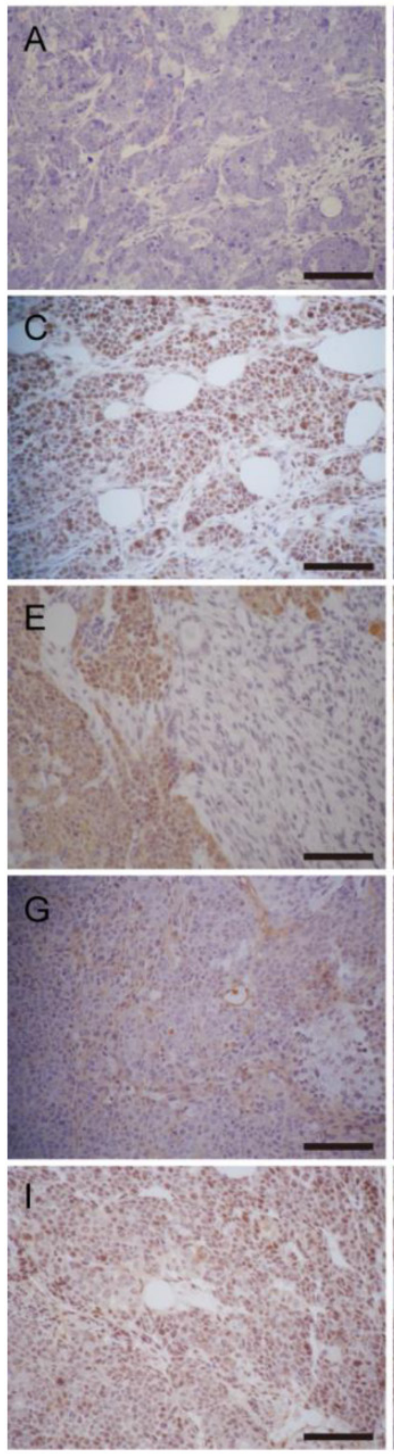

Figure 5. Tumor allografts generated by primary cells display a similar histologic phenotype to the parental tumor. H\&E staining of tumor sections to PT cell derived tumors (A) and DT cell derived tumors (B) show similar histologic phenotype. Comparable staining pattern of relative markers Ki67 (C and D), GFP (E and $F$ ), vimentin ( $G$ and $H)$, PPAR $\gamma 2$ ( $\mathrm{I}$ and $\mathrm{J}$ ) shows tumors developed from the two cell lines share similar properties. Scale bars: $100 \mu \mathrm{m}$.

We then assessed if the heterogeneous cells within the adipocyte lineage were derived by differentiation from the GFP positive cells. Spheroids were induced to adipocyte differentiation by addition of insulin and dexamethasone in the adherent culture condition. Differentiation into adipocytes was evaluated by Oil Red O staining (Fig. 6E). We found an accumulation of a significant number of fat droplets in both the miPS-LLCevPT and miPS-LLCevDT cells. These findings demonstrate the potential for adipocyte differentiation by these cancer stem-like cells, and implicate a cellular hierarchy similar to that comprising the heterogeneity of liposarcomas derived from miPS-LLCev cells.
Several studies have shown that CSCs can give rise to endothelial cells, which contribute to tumor vascularization thereby facilitating tumor growth [19, 36]. Since a population of $\mathrm{CD} 34^{+}$cells is considered to be a progenitor population of endothelial cells [37], we applied in vitro tube formation assay to evaluate the endothelial differentiation capacity of the miPS-LLCevPT and miPS-LLCevDT cells (Fig. 6D). The results indicate that cells formed vessel-like structures that contained a CD31 positive population of cells, which is a marker of differentiated endothelial cells, demonstrating that miPS-LLCevPT and miPS-LLCevDT cells can differentiate into mature endothelial cells [36]. Along with the adipocyte differentiation, these results validate the multipotency of both the miPS-LLCevPT and miPS-LLCevDT cells. Taken together, with tumorigenicity and self-renewal capacity, we conclude that the miPS-LLCev, miPS-LLCevPT and miPS-LLCevDT cells are CSCs capable of developing a liposarcoma that exhibit phenotypic heterogeneity.

\section{The invasive capacity of miPS-LLCev cells}

Although we subcutaneously transplanted miPS-LLCev cells into immunodeficient mice, the miPS-LLCevDT cells were established from tumor cells that had disseminated to the abdominal cavity in the mesentery. This suggests that a subpopulation of cells might already exist in the transplanted miPS-LLCev cells that possess a high metastatic ability, which is one characteristic of CSCs [1]. To address this possibility, we examined the invasive capacity of miPS-LLCev, miPS-LLCevPT and miPS-LLCevDT cells in vitro (Fig. 6F). Cells were seeded onto Matrigel-coated transwell membranes and after $30 \mathrm{~h}$, cells that invaded to the lower side of the filter were counted. Compared to the parental miPS-LLCev cells, the invasive capacities of both the miPS-LLCevPT and miPS-LLCevDT cells were significantly higher. This result suggests that cells with higher invasive ability are enriched during in vivo tumor development and those cells can metastasize.

\section{Discussion}

The present study demonstrates for the first time that cancer cell derived extracellular vesicles can facilitate the reprograming of differentiating miPSCs to convert into CSCs. The reprogrammed miPSCs, termed miPS-LLCev cells, exhibited sphere formation in suspension culture, tumorigenic capacity and metastasis to the abdominal cavity in immunodeficient mice. We also demonstrated the tumorigenicity and multipotency of the two cell lines that were established from primary tumors and disseminated tumors, termed miPS-LLCevPT and miPS-LLCevDT, 
with respect to their ability to differentiate into adipocyte and vascular endothelial cells, and that lead to the development of liposarcomas composed of a heterogeneous cellular population. Liposarcomas are the most common type of soft tissue sarcomas and are classified into five major groups, well-differentiated liposarcoma, dedifferentiated liposarcoma, myxoid liposarcoma, pleomorphilic liposarcoma, and mixed-type liposarcoma [28]. Pathological features of the miPS-LLCev tumors suggested that the tumors derived from miPS-LLCev cells were dedifferentiated liposarcomas. Similar pathological features were observed in secondary tumors that were derived from either miPS-LLCevPT or miPS-LLCevDT cells. Thus, we suggest that miPS-LLCev, miPS-LLCevPT and miPS-LLCevDT cells are potential models of liposarcoma CSCs.

The LLC derived tEVs are apparently affecting the differentiation potential of miPSCs, since we

A

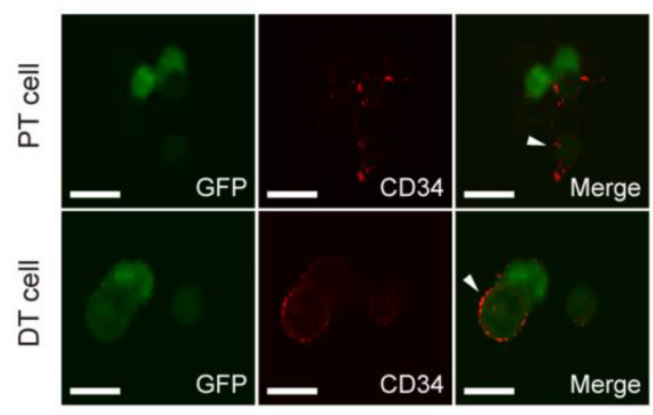

C

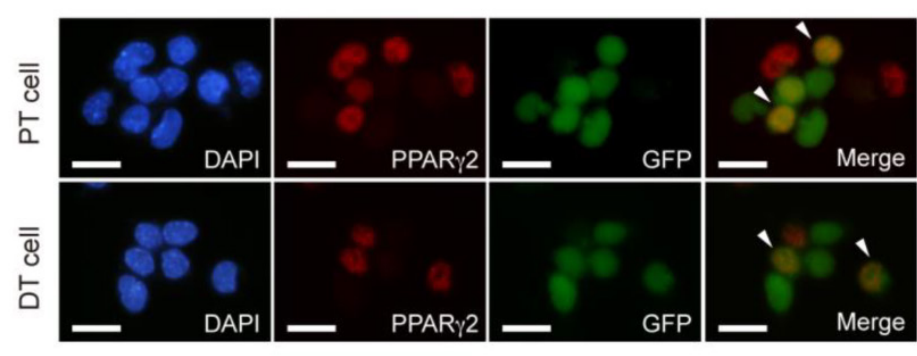

D

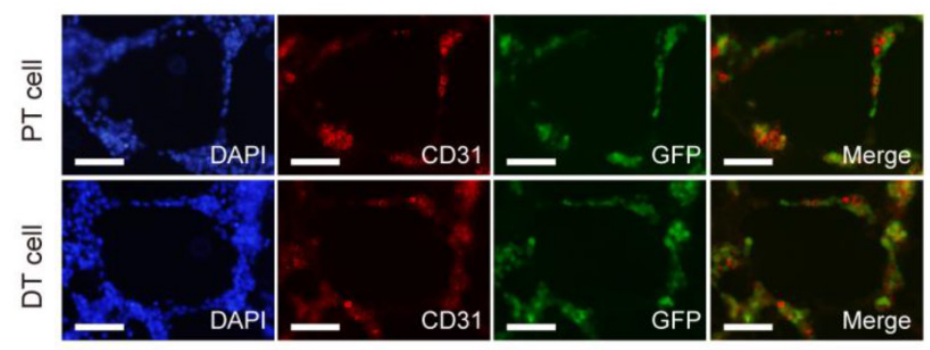

started the tEV treatment three days after removing LIF from the miPSCs culture when GFP fluorescence indicative of Nanog expression was not detected. Fujimori et al. has recently reported that the differentiation of ES cells in the presence of newborn bovine serum in vitro results in the generation of cells with CSC properties [2]. They failed to observe a similar effect in fetal bovine serum or adult bovine serum. Their results suggest that aberrant microenvironmental factors can reprogram normal stem cells through p53 regulation into CSCs. It has also been reported that tumor cells can inhibit p53 induction in adjacent host stromal cell fibroblasts [38]. Although we cannot rule out such a possibility for the effect of tEVs to directly regulate a subpopulation of stem cells after three days differentiation that are predisposed to giving rise to a CSC-like population, it is still possible that $\mathrm{EVV}$ may function in this capacity.

\section{B}

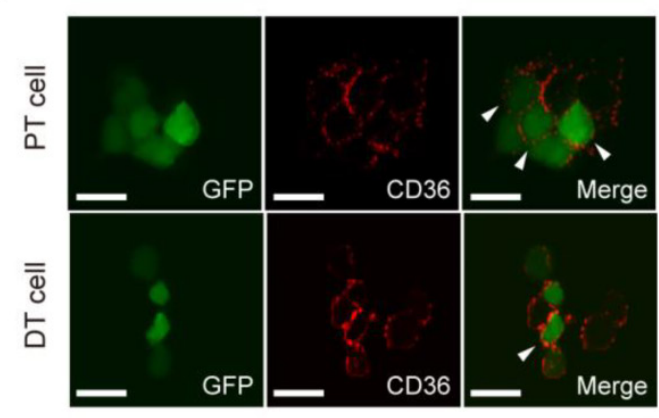

E

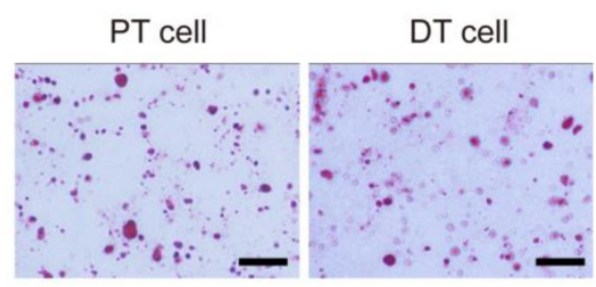

$\mathbf{F}$

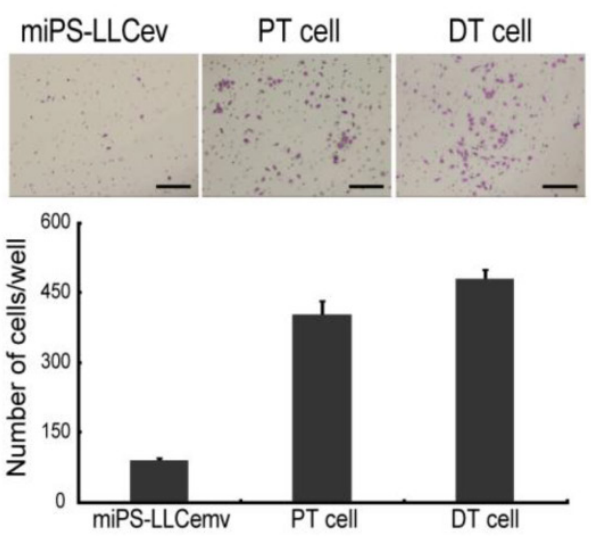

Figure 6. Cells derived from tumors possess heterogeneous populations. CD34 (A), CD36 (B), PPAR 2 (C) immunofluorescence staining of primary cells show different stages of adipocyte differentiation in primary bulk cell culture. Arrowheads are showing the double positive cells. (D) In virto tube formation assay shows both PT cell and DT cells possess tube formation ability. The CD3I positive and negative cells indicate the heterogeneity of primary cells. (E) Oil Red O staining after in vitro differentiation of spheroid cells, shows the two primary cell lines could differentiate into adipocytes. (F) Matrigel invasion assay shows significantly higher invasive capacity of both primary cells than resultant cell. DT cell line is slightly more invasive than the PT cell line. A, B and C scale bars: I0 $\mu \mathrm{m}$. D and E scale bars: $100 \mu \mathrm{m}$. F scale bar: $200 \mu \mathrm{m}$. 
tEVs can deliver various RNAs or oncoproteins to distant cells and thereby alter the translational profiles in these recipient cells [12, 20,39]. tEVs may also interact directly through surface proteins or receptors to promote specific signaling pathways [40]. In this context, the tEVs are known to facilitate the intercellular transfer of information to recipient cells and therefore possibly induce or accelerate their transformation. Our results clearly support this scenario, whereby CSCs could arise from normal stem cells or progenitor cells by tEVs. Although it is currently unclear as to the molecular mechanism(s) that govern this conversion process, one possibility is that functional microRNAs may be transferred by tEVs from LLC to differentiating miPSCs and initiate their malignant transformation. A number of studies have demonstrated that microRNAs perform a very critical role in carcinogenesis by altering target gene translation and/or by the induction of epigenetic modifications of genes [41]. In addition, microRNAs are involved in the regulation of epithelial-mescenchymal transition, one of the critical events that facilitate cellular reprogramming to acquire CSC properties [42, 43]. Moreover, microRNAs are known to be actively secreted through small vesicles, such as exosomes or microvesicles [20]. In several cases of cancers, microRNA profiles in tEVs have been proposed to function as diagnostic cancer biomarkers [44]. Thus, it would be informative in future studies to investigate the microRNA profiles of LLC derived tEVs.

We also found that the expression of two significant iPS reprogramming genes, Klf4 and Sox2, were upregulated in miPS-LLCev, miPS-LLCevPT and miPS-LLCevDT when these cells were cultured as spheroids. Klf4 is primarily expressed in postmitotic or differentiated cells of the skin and in the gastrointestinal tract and functions in a variety of roles to control differentiation and proliferation through cell cycle regulators in a content-dependent manner [45]. Recent studies have also reported that Klf4 is an essential and an early regulator of adipogenesis and stimulates adipogenesis by regulating C/EBP $\beta$ [46], and that the expression of Klf4 can be activated by PPARY through directly binding to its promotor [47]. Since the miPS-LLCevPT and miPS-LLCevDT cells express PPARy, the expression of Klf4 could be, in part, related to adipocyte differentiation that is occurring in these tumor cells. In addition, Klf4 is also an essential reprograming factor for iPS cells [18], and is essential for the self-renewal of ES cells [48]. According to recent reports, Klf4 may also contribute to the self-renewal of some unipotent CSCs [32]. Although some reports have shown a tumor suppressor role for Klf4, our results implicate Klf4 as an oncogene.

Sox 2 is another essential core transcription factor for reprogramming iPSCs and is important in establishing early embryonic cell fate decisions [49]. A very recent report has shown that Sox2 is important in the lineage determination of osteo-adipo progenitors by induction of PPARY [50]. For Sox2, it has also been shown to be important in maintaining the self-renewal of tumor-initiating cells in breast carcinomas [7], osteosarcomas [31] and glioblastomas [51]. Riggi et al. recently demonstrated that, Sox2 is a key factor in the determination and regulation of Ewing sarcoma CSCs [30]. Given the fact that miPS-LLCcm cells expressing lower levels of Sox 2 and Klf4 developed adenocarcinomas in immunodeficient mice [16], CSCs in different tumors may be essentially unique in their self-renewal mechanisms but differ in their capacity to differentiate depending upon the contents of transcription factors.

In addition, our recent results indicate that miPS-LLCcm cells and their differentiated progenies, can create a niche in vitro, and this niche might regulate the self-renewal and differentiation capacities of miPS-LLCcm. tEVs and/or soluble factor(s) from potential different progenitor cells are in part, responsible for the commitment of differentiation lineages of miPS-LLCcm [19]. Therefore, both tEVs and soluble factor(s) in the CM from tumor cells contain a unique composition or signature of factors that compose the general cancerous niche that can differentially contribute to the genesis/maintenance of CSCs with different lineage commitments. It is also conceivable that different cancer progenitor cells are derived from a more primitive CSC after exposure to different environmental components.

Soluble or vesicle contained components produced in an aberrant environment, such as pre-malignant lesions, inflammation and cancer itself, could selectively reprogram normal somatic tissue stem cells or progenitors to a malignant stage. Somatic stem cells/differentiating progenitor cells residing in close proximity to tumor cells might be transformed into CSCs, as well, generating a new source of CSCs in tumors. In this regard, He et al. identified hepatocellular carcinoma progenitor cells (HcPCs) in premalignant lesions in chronically damaged liver [52]. HcPCs are not malignant when introduced into normal mice, but can give rise to carcinomas in livers sustaining chronic damage. Thus, in support of our hypothesis, this chronically damaged liver could be regarded as a cancerous niche. Indeed, our findings relevant to a cancerous niche provide a new insight into the potential origin of CSCs and cancer.

\section{Abbreviations}

LLC: Lewis Lung Carcinoma; miPSCs: mouse induced pluripotent stem cells; CSCs: cancer stem 
cells; tEVs: tumor-derived extracellular vesicles; $\mathrm{CM}$ : conditioned medium; puro: puromycin; GFP: Green Fluorescent Protein; MEFs: mouse embryonic fibroblasts; LIF: leukemia inhibitory factor; ES: embryonic stem cell.

\section{Supplementary Material}

Additional File 1:

Supplementary Table S1, Supplementary Figures

S1-S4.

http://www.jcancer.org/v05p0572s1.pdf

\section{Acknowledgements}

This research has been performed under the Grant-in-Aid for Challenging Exploratory Research No. 23650598 (M.S.) and the Grant-in-Aid for Scientific Research (C) No. 24501315 (A.M.), Japan Society for Promotion of Science (JSPS).

\section{Competing Interests}

The authors have declared that no competing interest exists.

\section{References}

1 Li L and Neaves WB. Normal stem cells and cancer stem cells: the niche matters. Cancer Res. 2006;66:4553-7.

2 Fujimori $\mathrm{H}$, Shikanai M, Teraoka $\mathrm{H}$, et al. Induction of cancerous stem cells during embryonic stem cell differentiation. J Biol Chem. 2012;287:36777-91.

3 Clarke MF, Dick JE, Dirks PB, et al. Cancer stem cells--perspectives on current status and future directions: AACR Workshop on cancer stem cells. Cancer Res. 2006;66:9339-44

4 Galli R, Binda E, Orfanelli U, et al. Isolation and characterization of tumorigenic, stem-like neural precursors from human glioblastoma. Cancer Res. 2004;64:7011-21.

5 Eramo A, Lotti F, Sette G, et al. Identification and expansion of the tumorigenic lung cancer stem cell population. Cell Death Differ. 2008;15:504-14.

6 Suva ML, Riggi N, Stehle JC, et al. Identification of cancer stem cells in Ewing's sarcoma. Cancer Res. 2009;69:1776-81.

7 Leis $\mathrm{O}$, Eguiara A, Lopez-Arribillaga $\mathrm{E}$, et al. Sox2 expression in breast tumours and activation in breast cancer stem cells. Oncogene. 2012;31:1354-65.

8 Camussi G, Deregibus MC, Bruno S, et al. Exosomes/microvesicles as a mechanism of cell-to-cell communication. Kidney Int. 2010;78:838-48

9 Raposo SJGaG. As we wait: coping with an imperfect nomenclature for extracellular vesicles. Journal of Extracellular Vesicles. 2013;2:20389.

10 Wysoczynski $\mathrm{M}$ and Ratajczak MZ. Lung cancer secreted microvesicles: underappreciated modulators of microenvironment in expanding tumors. Int J Cancer. 2009;125:1595-603.

11 Peinado H, Aleckovic M, Lavotshkin S, et al. Melanoma exosomes educate bone marrow progenitor cells toward a pro-metastatic phenotype through MET. Nat Med. 2012;18:883-91.

12 Al-Nedawi K, Meehan B, Micallef $\mathrm{J}$, et al. Intercellular transfer of the oncogenic receptor EGFRvIII by microvesicles derived from tumour cells. Nat Cell Biol. 2008:10:619-24.

13 Kalluri $\mathrm{R}$ and Zeisberg M. Fibroblasts in cancer. Nat Rev Cancer. 2006;6:392-401.

14 Castellana D, Zobairi F, Martinez MC, et al Membrane microvesicles as actors in the establishment of a favorable prostatic tumoral niche: a role for activated fibroblasts and CX3CL1-CX3CR1 axis. Cancer Res. 2009;69:785-93.

15 Cho JA, Park H, Lim EH, et al. Exosomes from breast cancer cells can convert adipose tissue-derived mesenchymal stem cells into myofibroblast-like cells. Int J Oncol. 2012;40:130-8.

16 Chen L, Kasai T, Li Y, et al. A model of cancer stem cells derived from mouse induced pluripotent stem cells. PLoS One. 2012;7:e33544.

17 Muralidharan-Chari V, Clancy JW, Sedgwick A, et al. Microvesicles: mediators of extracellular communication during cancer progression. J Cell Sci. 2010;123:1603-11.

18 Okita K, Ichisaka T and Yamanaka S. Generation of germline-competent induced pluripotent stem cells. Nature. 2007;448:313-7.

19 Matsuda S, Yan T, Mizutani A, et al. Cancer stem cells maintain a hierarchy of differentiation by creating their niche. Int J Cancer. 2014;135:27-36.
20 Valadi $\mathrm{H}$, Ekstrom $\mathrm{K}$, Bossios A, et al. Exosome-mediated transfer of mRNAs and microRNAs is a novel mechanism of genetic exchange between cells. Nat Cell Biol. 2007;9:654-9.

21 Ben-Porath I, Thomson MW, Carey VJ, et al. An embryonic stem cell-like gene expression signature in poorly differentiated aggressive human tumors. Nat Genet. 2008;40:499-507.

$22 \mathrm{Yu} \mathrm{S}$, Liu C, Su K, et al. Tumor exosomes inhibit differentiation of bone marrow dendritic cells. J Immunol. 2007;178:6867-75.

23 Miyoshi N, Ishii H, Sekimoto M, et al. Properties and identification of cancer stem cells: a changing insight into intractable cancer. Surg Today. 2010;40:608-13

24 Takahashi K and Yamanaka S. Induction of pluripotent stem cells from mouse embryonic and adult fibroblast cultures by defined factors. Cell. 2006;126:663-76.

25 Niwa H, Miyazaki J and Smith AG. Quantitative expression of Oct-3/4 defines differentiation, dedifferentiation or self-renewal of ES cells. Nat Genet. 2000;24:372-6.

26 Chang CC, Shieh GS, Wu P, et al. Oct-3/4 expression reflects tumor progression and regulates motility of bladder cancer cells. Cancer Res. 2008;68:6281-91.

27 Lehrke $\mathrm{M}$ and Lazar MA. The many faces of PPARgamma. Cell. 2005;123:993-9.

28 Dodd LG. Update on liposarcoma: a review for cytopathologists. Diagn Cytopathol. 2012;40:1122-31.

29 Horvai AE, Schaefer JT, Nakakura EK, et al. Immunostaining for peroxisome proliferator gamma distinguishes dedifferentiated liposarcoma from other retroperitoneal sarcomas. Mod Pathol. 2008;21:517-24.

30 Riggi N, Suva ML, De Vito C, et al. EWS-FLI-1 modulates miRNA145 and SOX2 expression to initiate mesenchymal stem cell reprogramming toward Ewing sarcoma cancer stem cells. Genes Dev. 2010;24:916-32.

31 Basu-Roy U, Seo E, Ramanathapuram L, et al. Sox2 maintains self renewal of tumor-initiating cells in osteosarcomas. Oncogene. 2012;31:2270-82.

32 Leng Z, Tao K, Xia Q, et al. Kruppel-like factor 4 acts as an oncogene in colon cancer stem cell-enriched spheroid cells. PLoS One. 2013;8:e56082.

33 Zhang Y, Young ED, Bill K, et al. Heterogeneity and immunophenotypic plasticity of malignant cells in human liposarcomas. Stem Cell Res. 2013;11:772-81.

34 Daquinag AC, Zhang Y, Amaya-Manzanares F, et al. An isoform of decorin is a resistin receptor on the surface of adipose progenitor cells. Cell Stem Cell. 2011;9:74-86.

35 Mechtersheimer G. Towards the phenotyping of soft tissue tumours by cell surface molecules. Virchows Arch A Pathol Anat Histopathol. 1991;419:7-28.

36 Wang R, Chadalavada K, Wilshire J, et al. Glioblastoma stem-like cells give rise to tumour endothelium. Nature. 2010;468:829-33.

37 Fina L, Molgaard HV, Robertson D, et al. Expression of the CD34 gene in vascular endothelial cells. Blood. 1990;75:2417-26.

38 Bar J, Feniger-Barish R, Lukashchuk N, et al. Cancer cells suppress p53 in adjacent fibroblasts. Oncogene. 2009;28:933-6.

39 Skog J, Wurdinger T, van Rijn S, et al. Glioblastoma microvesicles transport RNA and proteins that promote tumour growth and provide diagnostic biomarkers. Nat Cell Biol. 2008;10:1470-6.

40 Pluskota E, Woody NM, Szpak D, et al. Expression, activation, and function of integrin alphaMbeta2 (Mac-1) on neutrophil-derived microparticles. Blood. 2008;112:2327-35.

41 Zhang P, Bill K, Liu J, et al. MiR-155 is a liposarcoma oncogene that targets casein kinase-1alpha and enhances beta-catenin signaling. Cancer Res. 2012;72:1751-62.

42 Gregory PA, Bracken CP, Bert AG, et al. MicroRNAs as regulators of epithelial-mesenchymal transition. Cell Cycle. 2008;7:3112-8.

43 Mani SA, Guo W, Liao MJ, et al. The epithelial-mesenchymal transition generates cells with properties of stem cells. Cell. 2008;133:704-15.

44 Rabinowits G, Gercel-Taylor C, Day JM, et al. Exosomal microRNA: a diagnostic marker for lung cancer. Clin Lung Cancer. 2009;10:42-6.

45 Garrett-Sinha LA, Eberspaecher H, Seldin MF, et al. A gene for a novel zinc-finger protein expressed in differentiated epithelial cells and transiently in certain mesenchymal cells. J Biol Chem. 1996;271:31384-90.

46 Birsoy K, Chen $\mathrm{Z}$ and Friedman J. Transcriptional regulation of adipogenesis by KLF4. Cell Metab. 2008;7:339-47.

$47 \mathrm{Li} \mathrm{S}$, Zhou Q, He H, et al. Peroxisome proliferator-activated receptor gamma agonists induce cell cycle arrest through transcriptional regulation of Kruppel-like factor 4 (KLF4). J Biol Chem. 2013;288:4076-84.

$48 \mathrm{Li} \mathrm{Y,} \mathrm{McClintick} \mathrm{J,} \mathrm{Zhong} \mathrm{L,} \mathrm{et} \mathrm{al.} \mathrm{Murine} \mathrm{embryonic} \mathrm{stem} \mathrm{cell} \mathrm{differentiation} \mathrm{is}$ promoted by SOCS-3 and inhibited by the zinc finger transcription factor Klf4. Blood. 2005;105:635-7.

49 Avilion AA, Nicolis SK, Pevny LH, et al. Multipotent cell lineages in early mouse development depend on SOX2 function. Genes Dev. 2003;17:126-40.

50 Seo E, Basu-Roy U, Gunaratne PH, et al. SOX2 regulates YAP1 to maintain stemness and determine cell fate in the osteo-adipo lineage. Cell Rep. 2013;3:2075-87.

51 Gangemi RM, Griffero F, Marubbi D, et al. SOX2 silencing in glioblastoma tumor-initiating cells causes stop of proliferation and loss of tumorigenicity. Stem Cells. 2009;27:40-8.

52 He G, Dhar D, Nakagawa $H$, et al. Identification of liver cancer progenitors whose malignant progression depends on autocrine IL-6 signaling. Cell. 2013;155:384-96 